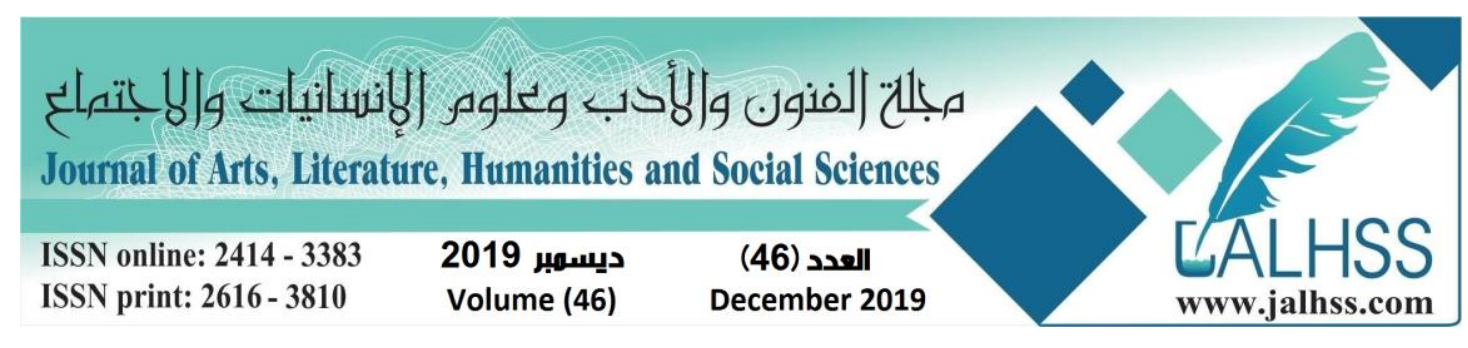

\title{
تقويم كتاب العلوم (الجزء الأول) للصف الثاني متوسط في ضوء التربية الجمالية المية
}

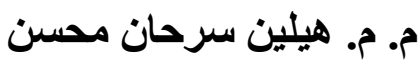
م.م. جمفر فائز كاظم

المديرية العامة لتربية القادسيةـ وزارة التربية ـ العراق

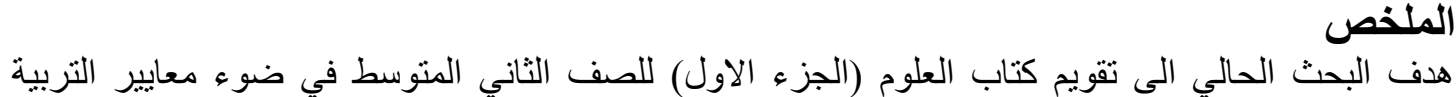

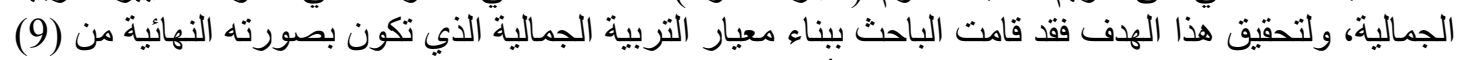

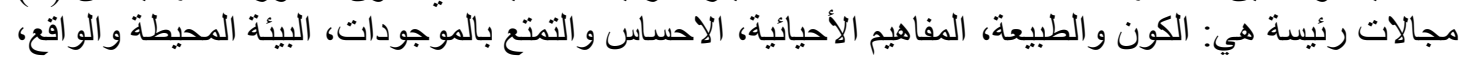

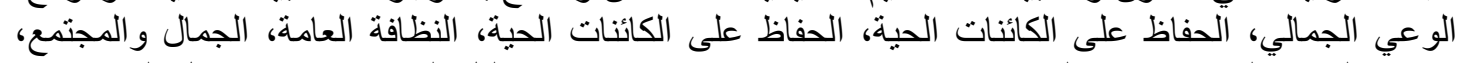

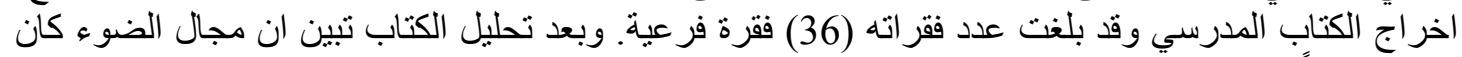

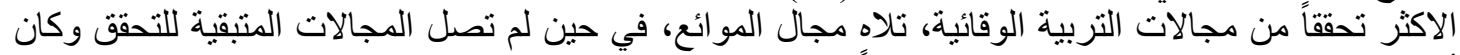

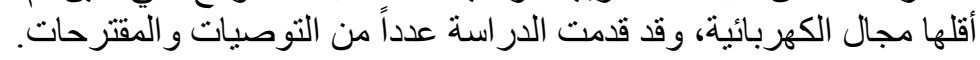

\section{Assessment of The Book of Science (Part I) for The Second Grade Medium in The Light of Aesthetic Education}

\author{
Helen Sarhan Mohsen \\ Jafar Fayez Kazem
}

\begin{abstract}
The aim of the present research is to evaluate the book of science (first part) for the second intermediate grade in the light of the standards of aesthetic education. With the assets, the surrounding environment and reality, aesthetic awareness, preservation of living organisms, preservation of living organisms, public hygiene, beauty and society, the output of the textbook has reached the number of paragraphs (36) subparagraph. After analyzing the book, it was found that the field of light was the most realized from the fields of preventive education, followed by the field of fluids, while the remaining areas did not reach the verification and the least was the field of electrical, the study has made a number of recommendations and proposals.
\end{abstract}




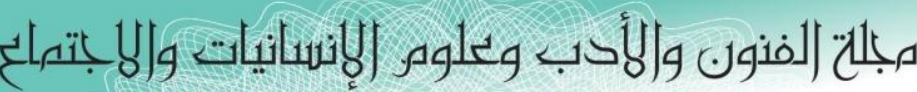
Journal of Arts, Literature, Humanities and Social Sciences

ISSN online: 2414 - 3383

ISSN print: 2616 - 3810

\section{9 ديسموبر Volume (46)}

\section{(46) العدد}

December 2019

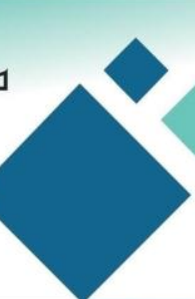

LALHSS

www.jalhss.com

مشكلة البحث: - مثئ

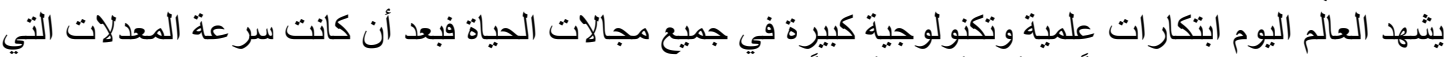

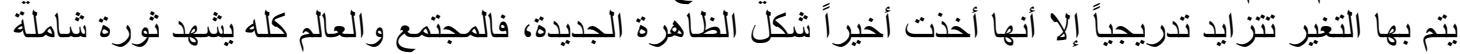

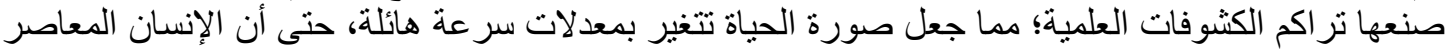

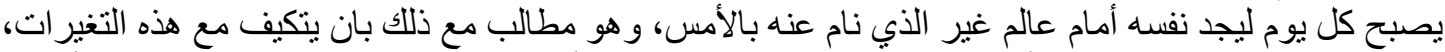

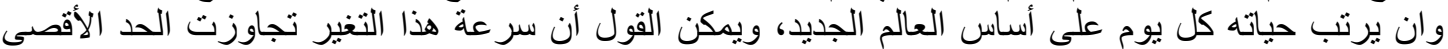

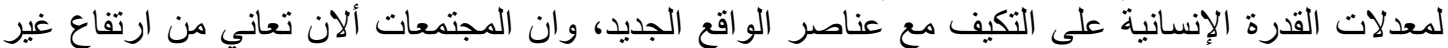

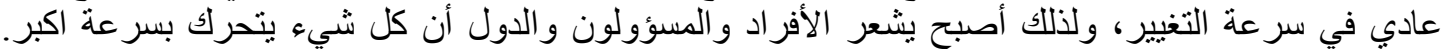

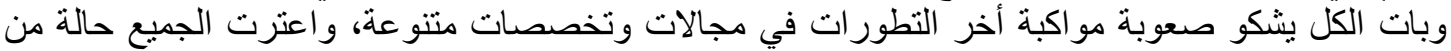

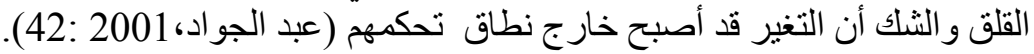

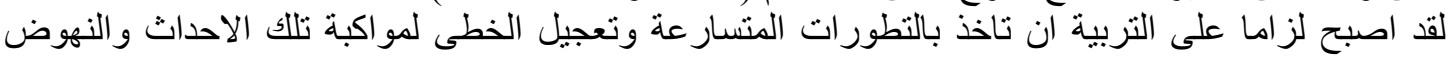

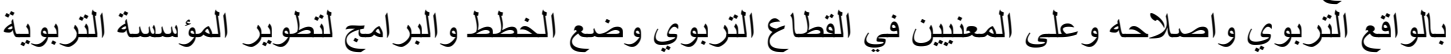

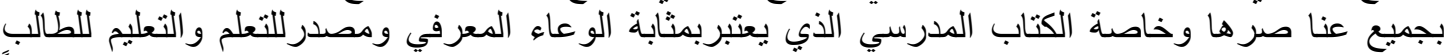

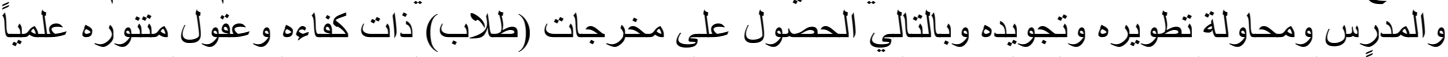

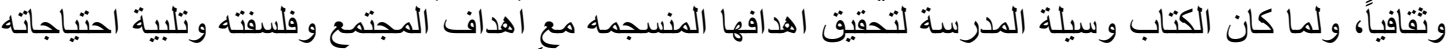

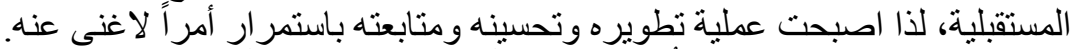

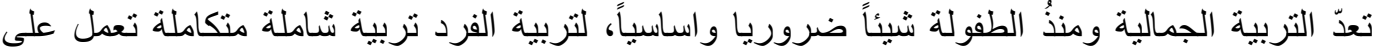

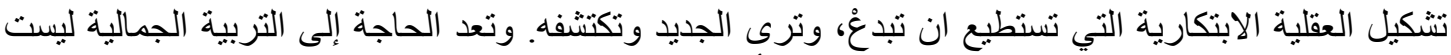

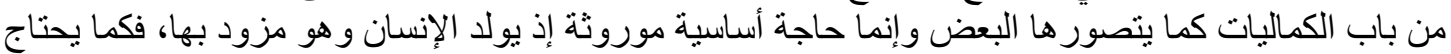

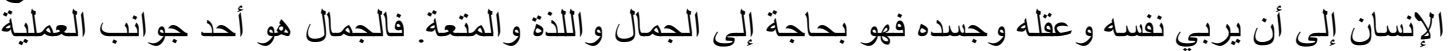

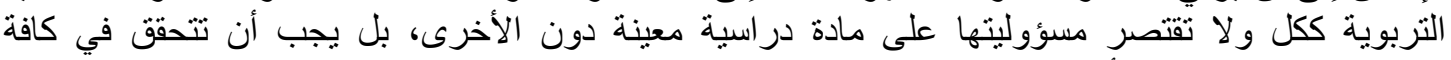

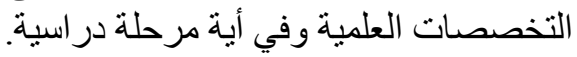

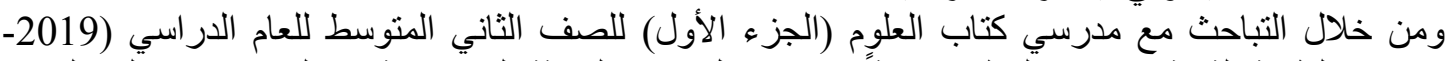

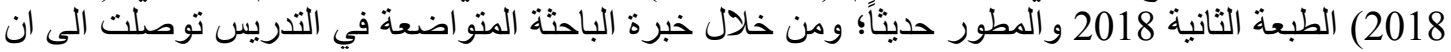

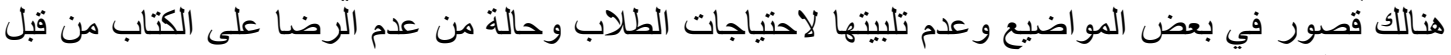

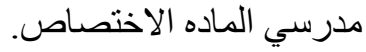
ان هذه الدراسة تبحث في تقويم كتاب العلوم (الجزء الأول) للصف الثاني المتوسط في ضوء الأه معايير التربية

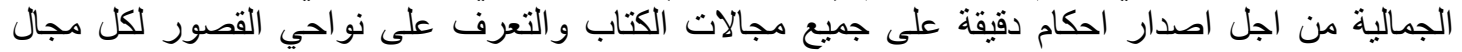

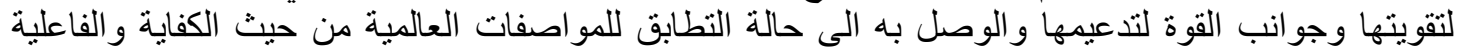

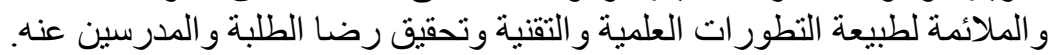

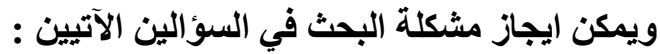

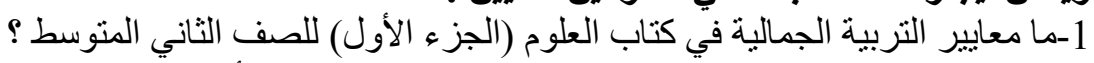

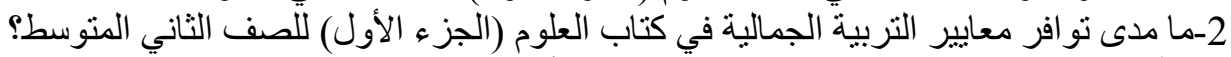

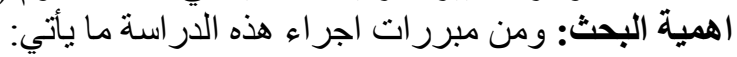

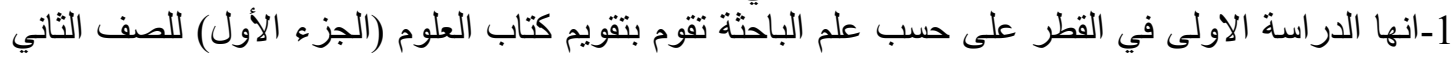
المنوسط في ضو الهو معايير التربية الجمالية.

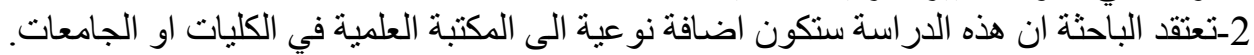

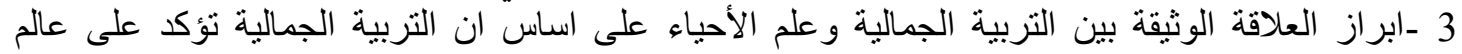

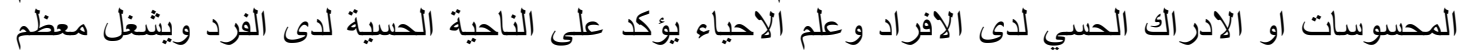
حواسه.

4ـاعتقاداً من الباحثة ان هذا البحث قد يفيد المعنيين في تأليف الكتب و المناهج في وزارة التربية.

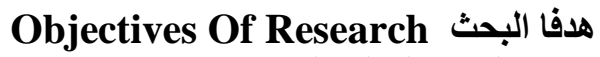

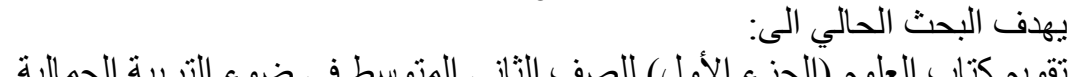




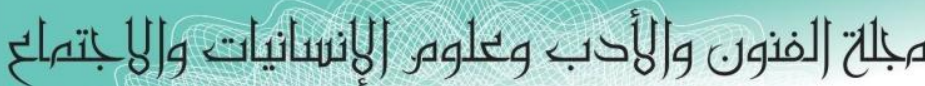

Journal of Arts, Literature, Humanities and Social Sciences

ISSN online: 2414 - 3383

ISSN print: $\mathbf{2 6 1 6}-\mathbf{3 8 1 0}$

\section{9 دبسمبر \\ Volume (46)}

\section{(46) العدد}

December 2019

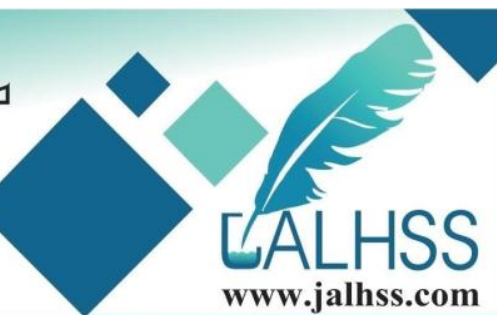

حدود البحث: Limitation The Research

1- كتاب العلوم (الجزء الأول) للصف الثاني المتوسط الطبعة الثانية 2018

تحديد المصطاح:

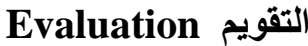

هو عملية إصدار حكم على أهمية وكفاءة الثيء المقاس، وبني هذا الحكم على أساس بيانات مستخرجة

من درجات الاختبار.

Aesthetic education التربية الجمالية

عرفها أبو حطب 1973 بأنها: النشاط الذي يهدف إلى تتمية الانسان في مختلف مر احل حياته متمتعاً

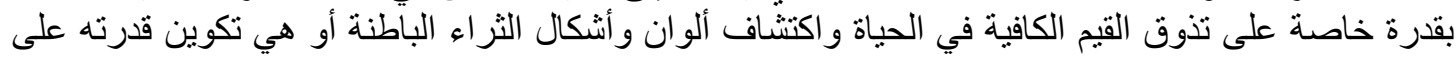

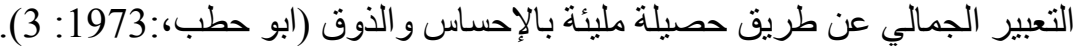

تعرف الباحثة التربية الجمالية :

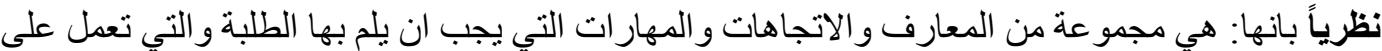

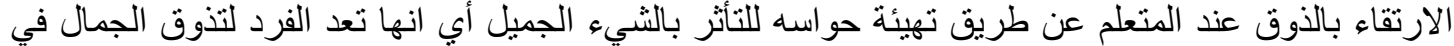

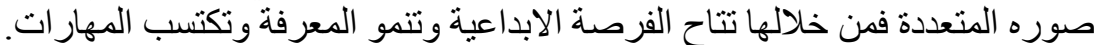

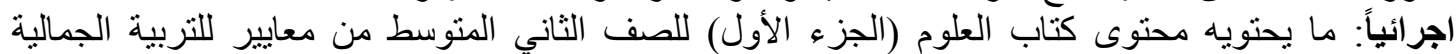

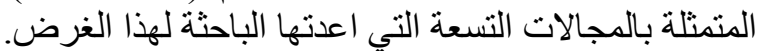

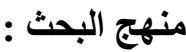

فقد عرف برنارد بيرلسون المشار اليه في (عبد الحميد1 1983) تحليل المحتوى بانه: أسلوب البحث الذي يهدف

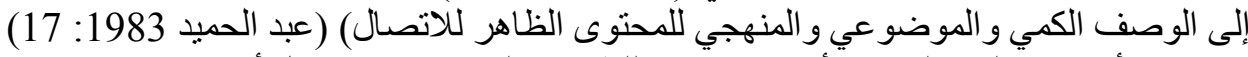

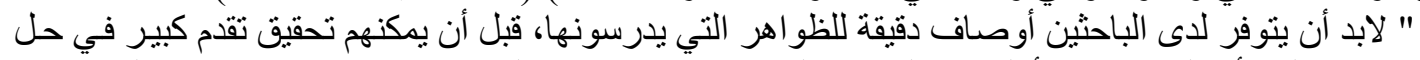

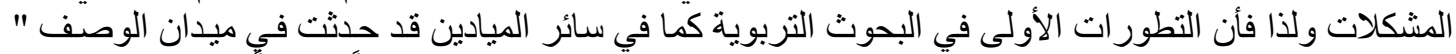

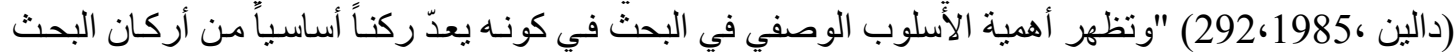

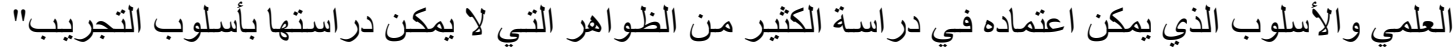

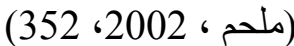

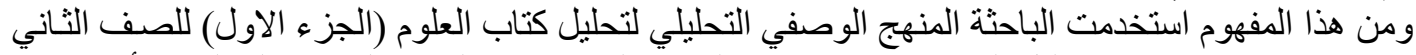

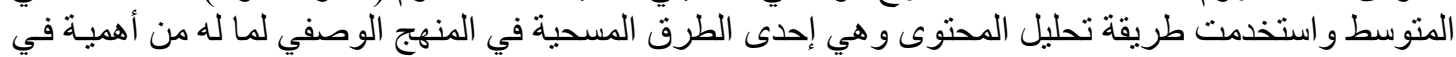

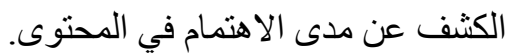

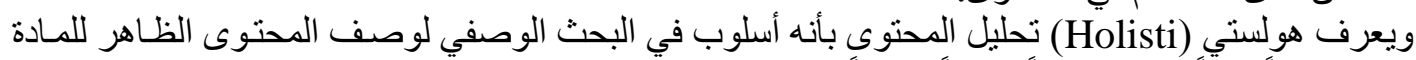

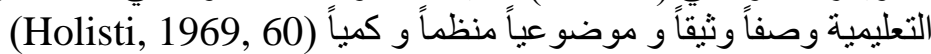

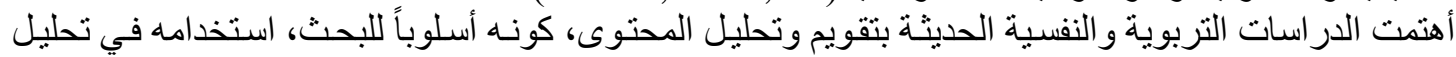

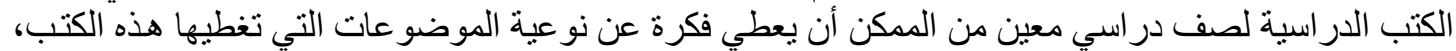

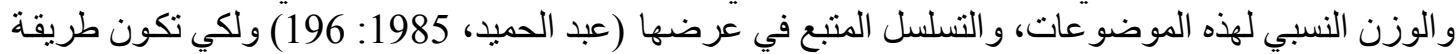

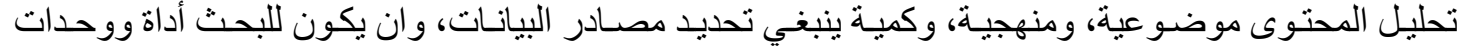

تحليل ووحدات تعداد وقو اعد صريحة وو اضحة لطريقة التحليل (Banks,1971,95). تعديد مصادر البيانات : تصنيل

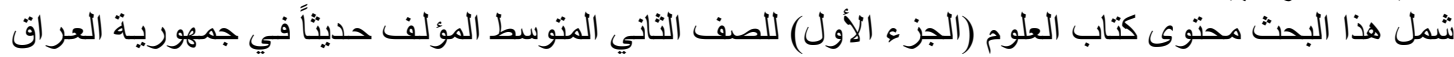
للعام الدر اسي 2017-

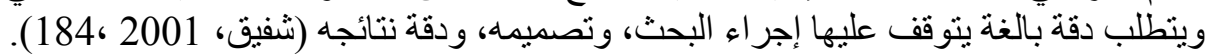

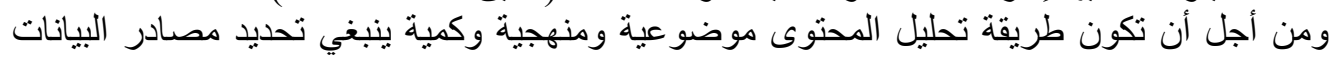

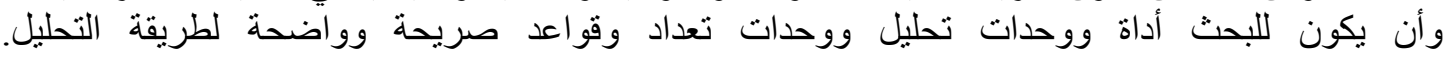

.(Banks, 1971: 95) 


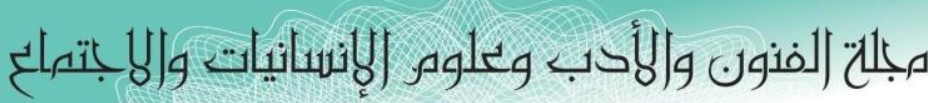
Journal of Arts, Literature, Humanities and Social Sciences

ISSN online: 2414 - 3383

ISSN print: $\mathbf{2 6 1 6}-\mathbf{3 8 1 0}$

\section{ديسمة 2019 \\ Volume (46)}

العدد (46)

December 2019

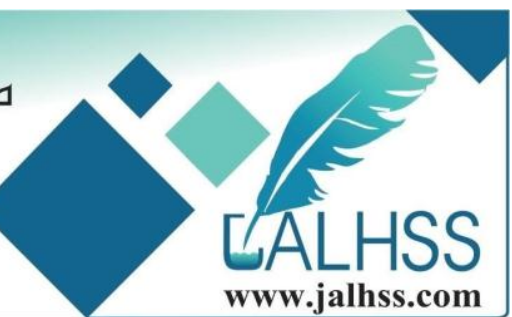

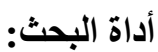

يعد بناء اداة البحث من الاجراءات المهمة في عملية تحليل المحتوى لأنها تساعد الباحثة على استيفاء عناصر

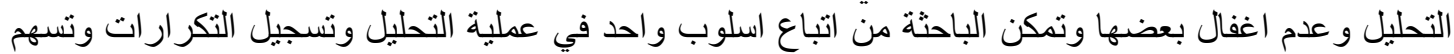
في التقليل من الوقت و المجهود الذي يبذل في عملية التحليل وتمكن الباحثة من تكميم البيانات (عطية،

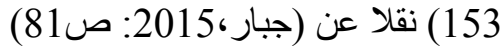

ولتحقيق هدف البحث تطلب بناء معيار اً للتربية الجمالية التي يفضل تضمينها في كتاب العلوم (الجزء الاول) للصف الثاني المتوسط، ليتم في ضوئه تحليل محتوى ذلك الكتاب وبيان مدى مر اعاته لتلكانك التربية الجمالية حيث التيث

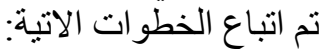

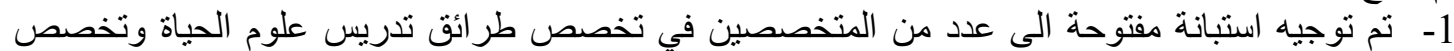
التربية الفنية وبعض التئ مشرفي الاحياء حول اهم مجالات التربية الجمالية التي يفضل تضمينها في كتاب العلوم

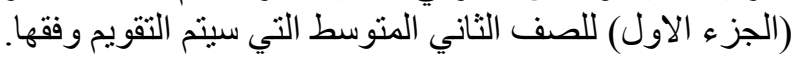

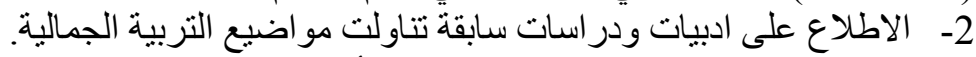

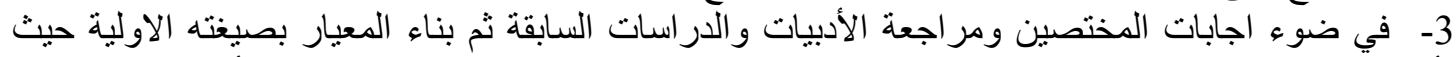

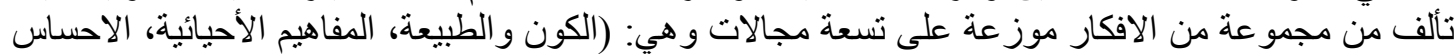

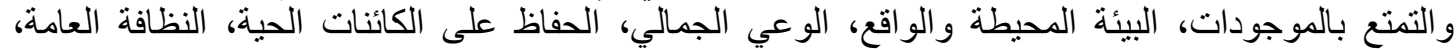
الجمال و المجتمع، اخر اج الكتاب المدرسي). وقد تألف المعيار بصورته الأولية من (36) فقرة فرعية.

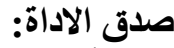
يمكن أن يعرف الصدق بأنه صلاحية الاداة المستعملة لقياس ما وضعت من أجله (Drost 2011: 114) ويقصد

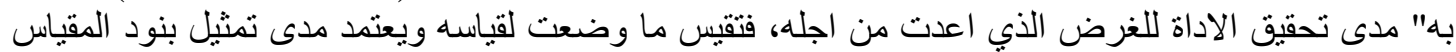
تمثيلا سليما للمجال الذي بر ادئ قلاد قياسه" (الكندي،2006: 215).

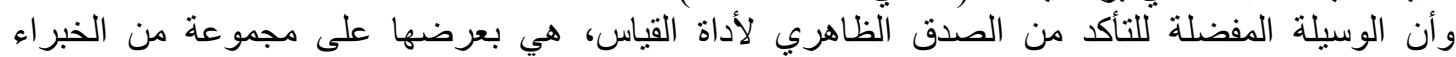

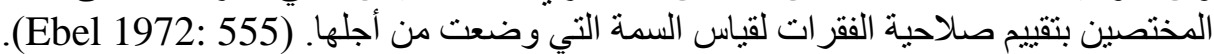

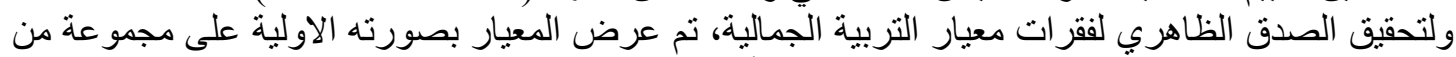

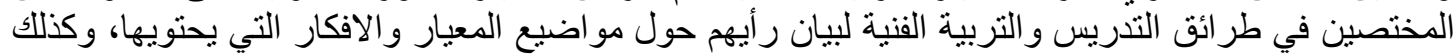

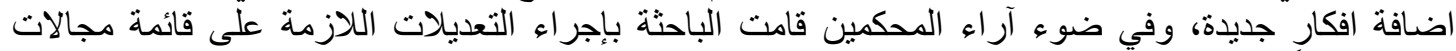

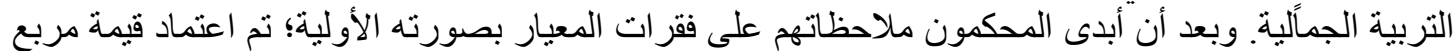

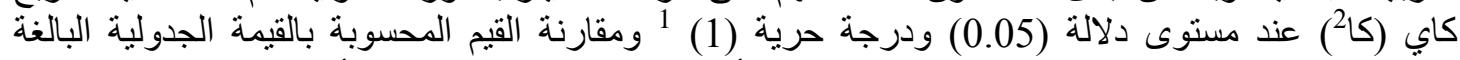

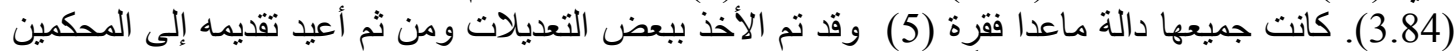

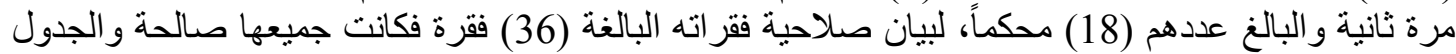
(1) يبين درجة إتفاق المحكمون على الفقرات باستعمال مربع كابي.

(1987) حسبت القيمة الجدولية لمربع كا2 عند درجة حرية (df) = (عدد الأعمدة -1) (عدد الصفوف-1). (أبو النيل

(230 -228:1987

$$
\begin{aligned}
1=(1-2)(1-2) & =\mathrm{df} \\
3.84 & =\alpha
\end{aligned}
$$




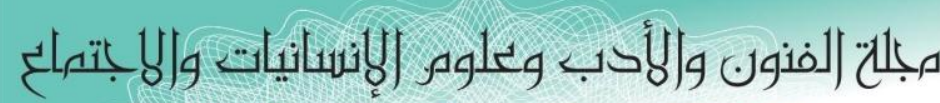
Journal of Arts, Literature, Humanities and Social Sciences

\begin{tabular}{|c|c|c|}
\hline $\begin{array}{l}\text { ISSN online: } 2414-3383 \\
\text { ISSN print: } 2616 \text { - } 3810\end{array}$ & ديسموبر 2019 & $\begin{array}{c}\text { العدد (46) } 2019 \\
\text { December }\end{array}$ \\
\hline
\end{tabular}

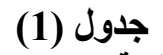

جدول استجابات المحكمين على فقرات المقياس وقيمة مريع كاي عند مستوى دلالة 0.05 ودرجة حرية 1 والبالغة 3.84

\begin{tabular}{|c|c|c|c|c|c|c|}
\hline القر ار & الدالة & الجدولية & قالمحسوبة 2 & الر افضين & المو افقي & تسلسل الفقرة \\
\hline تبقى كما هي & دالة & 3.84 & 18 & 0 & 18 & 15 ، 10، 4، 2، \\
\hline تبقى كما هي & دالة ل & 3.84 & 14.22 & 1 & 17 & 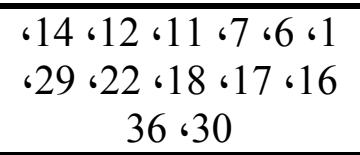 \\
\hline تنقى بعد & 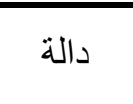 & 3.84 & 10.89 & 2 & 16 & 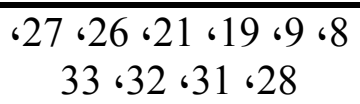 \\
\hline تلتقى بعد & 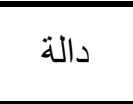 & 3.84 & 8 & 3 & 15 & 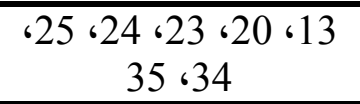 \\
\hline تستبدل & غير دالة & 3.84 & 3.56 & 5 & 13 & 5 \\
\hline
\end{tabular}

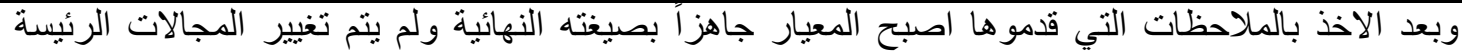

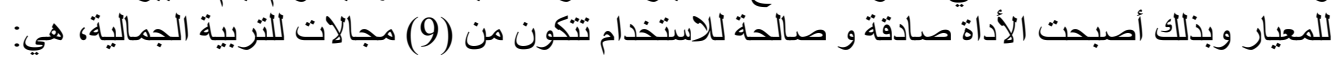
تحليل محتوى كتب علم الأحياء للمرحلة المتونة المتيطة: اعتمدت الباحثة في تحليل المحتوى على الاعلى الآتي:

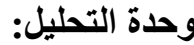 يستخدم عادة في تحليل المحتوى وحدتان هما:

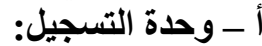

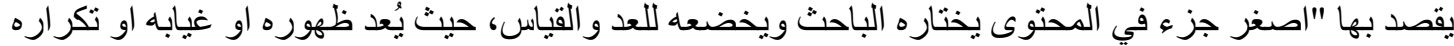

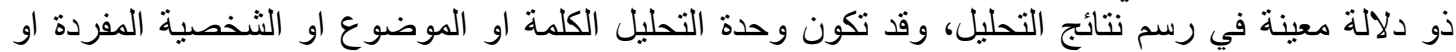

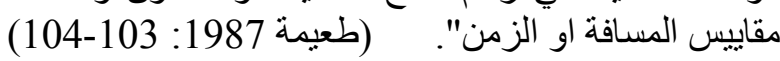

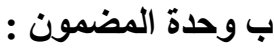

و هي الهيكل المحيط بوحدة التسجيل و ينبغي معرفته بغية الوصول إلى تشخيص وحدة التسجيل ( Stone

$$
\text { ج). (,1966,41 }
$$

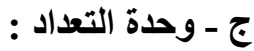

ويُستخدم التكرار وحدة للتعداد في حالة ظهور الفكرة التي تعبر عن فقرة من فقرات المعيار المستخدم ويُعطى

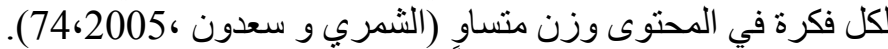
وفي هذا البحث فقد استعملت الباحثة الفكرة (them) كوحدة للتحليل لان لهان لها من السعة ما يكفي لإعطاء معنى

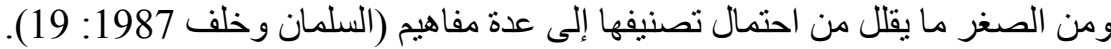
ويوجد نو عان من الفكرة هما: 1- الفكرة الصريحة: وهي الجملة او شبيهها التي يشار فيها صر احة وبشكل مباشر الرغبة في شيء او الرغبة

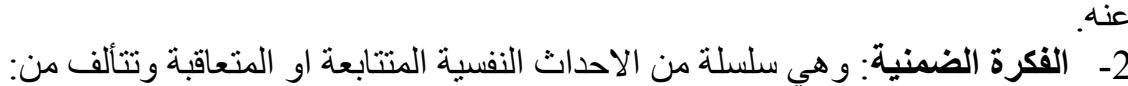

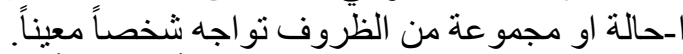
بـالسلوك الذي يستجيب به الفرد داخلياً وخارجياً. ج-نتائج ذللك السلوك سو اء كانت مادية او اجتماعية ام داخلية. (عبد الرحمن و عدنان،2007: 216) 


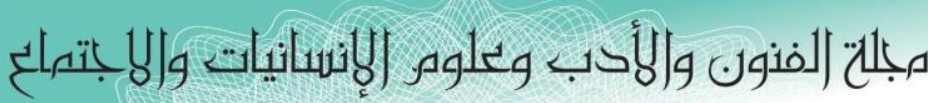
Journal of Arts, Literature, Humanities and Social Sciences

ISSN online: 2414 - 3383

ISSN print: 2616 - 3810

\section{9 ديسوة Volume (46)}

\section{العدد (46)} December 2019

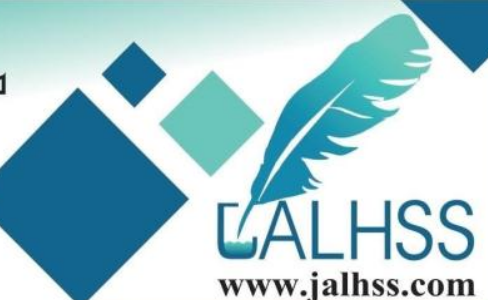

و استعملت الباحثة الفكرة الصريحة و الفكرة الضمنية بوصفهما وحدتي التسجيل باعتبار أن كتب علم الاحياء كتب

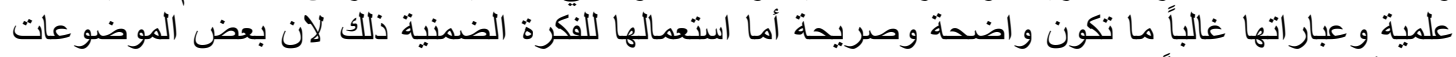

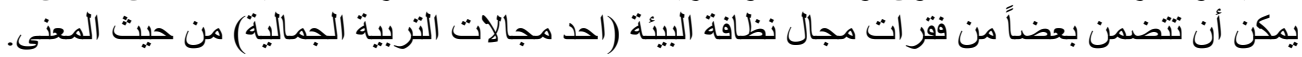

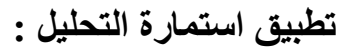

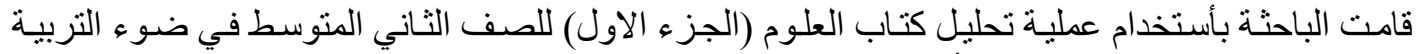

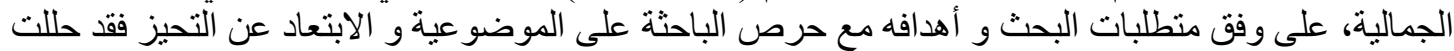

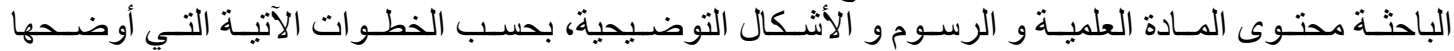

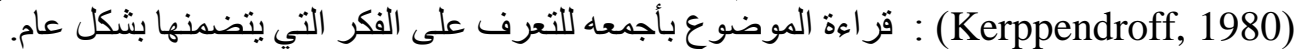

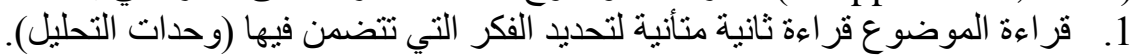

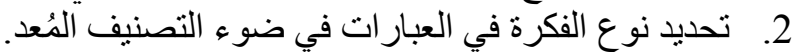
3. تفريغ نتائج التحليل في جداول التحليل المعدة لهذا الغرض في من اجل المعالجة الإحصائية. (Kerppendroff, 1980, 22)

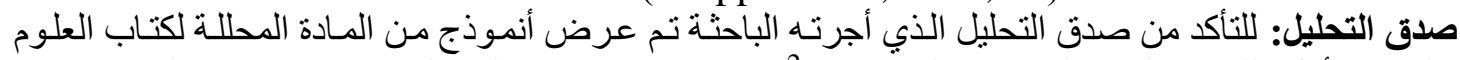

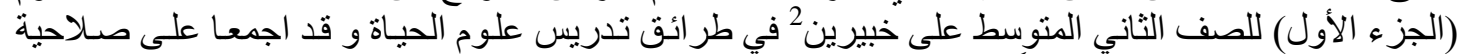

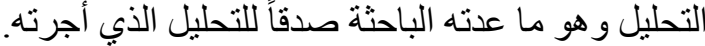

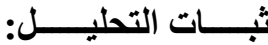

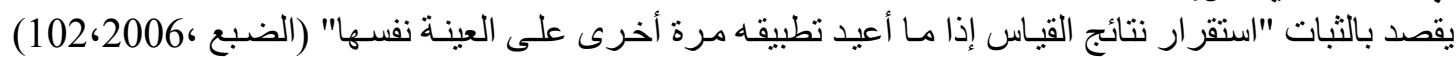

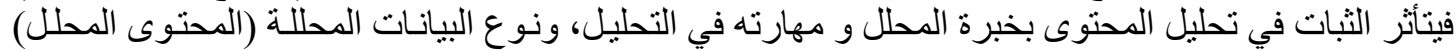

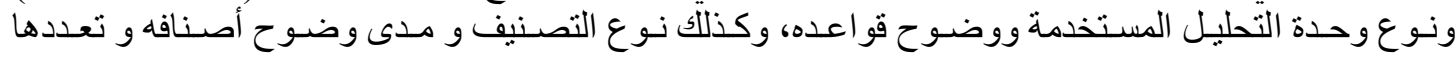

(Festinger, 1970, 27)

ولكي يكون التحليل موضو عياً و للحصول على ثبات مقبول ، استخدمت الباحثة نوعين من الثبات و هما:

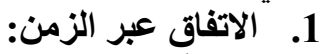
بمعنى أن يحصل عالزمل الملل أو مجموعة المحللين على النتائج نفسها إذا حللو ا على وفق تصنيف واحد للمحتوى

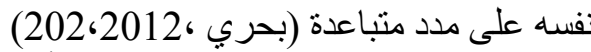

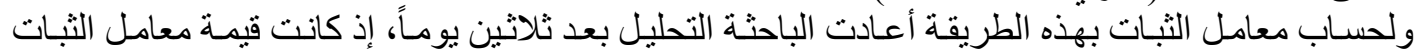
المحسوبة (0.94) باستعمال معادلة " هولستي "و هي قيمة مرتفعة، وتعكس درجة عالية من الثقة بنتائج البحث

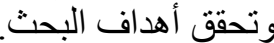

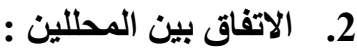

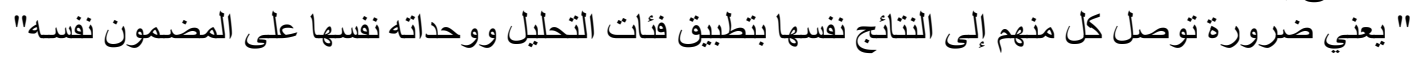

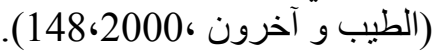

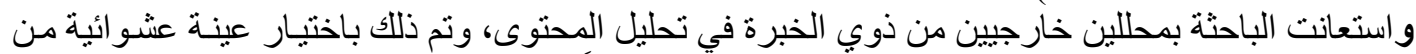

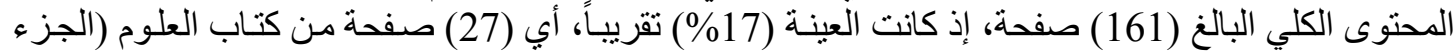

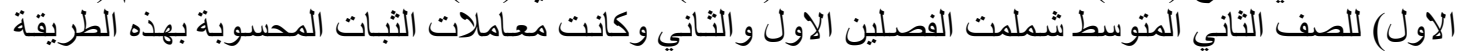

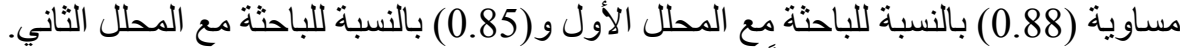

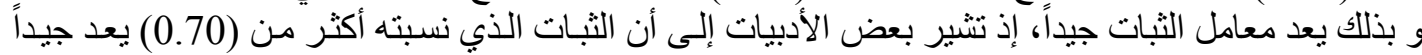
(Stmbly \& Kenneth, 1972,105) وييين الجدول (2) قيمة معاملات الثبات باستخدام معادلة هولستي. 


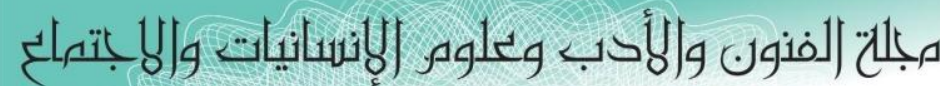

Journal of Arts, Literature, Humanities and Social Sciences

ISSN online: 2414 - 3383

ISSN print: $\mathbf{2 6 1 6}-\mathbf{3 8 1 0}$

\section{ديسوبر 2019 \\ Volume (46)}

العدد (46)

December 2019

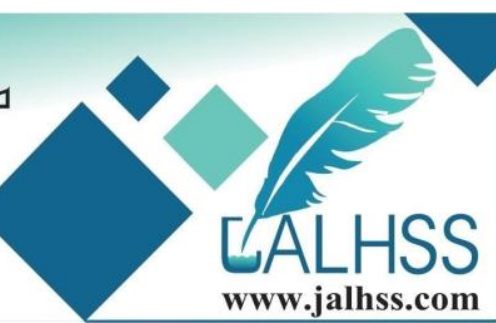

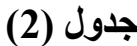

ق قيمة معساملات الثبات

\begin{tabular}{|c|c|c|}
\hline 0.94 & الباحثة بعد مرور30يوماً & الاتفاق عبر الزمن \\
\hline 0.88 & بين الباحثة والمحلل الأول & \multirow[t]{3}{*}{ الاتفات بين المحللين } \\
\hline 0.85 & بين الباحثة والمحلل الثاني & \\
\hline 0.91 & بين المحلل الأول والثاني & \\
\hline
\end{tabular}

تحديد النسبة المحكية لمقارنة نتائج التحليل:

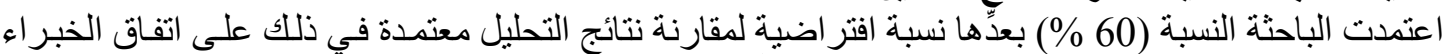

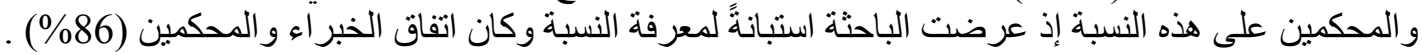
عرض التتائج وتفسيرها:

ـ الكثف عن تضمين محتوى كتاب العلوم (الجزءعالأول) للصف الثاني المتوسط لمجالات التربية الجمالية.

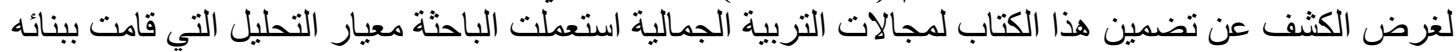
وفي ضوء التحليل الذي قامت بهاه، تعرض نتائج تحليل محتوى كتاب العلوم (الجزء الاول) للَّف الصف الثاني

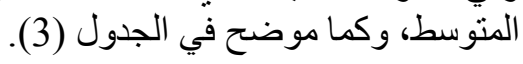

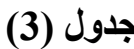

التكرارات والنسب المئوية لمجالات التربية الجمالية في كتاب العلوم (الجزء الاول) للصف المتوسط

\begin{tabular}{|c|c|c|c|c|c|}
\hline المتحقة المجال & الترتيب & للمجال النسبة المئوية & المجال عجوع تكر ارات & المجالات الرئيسة & $ت$ \\
\hline$\% 21.78$ & الخامس & $\% 8.43$ & 7 & الكون و الطبيعة & 1 \\
\hline$\% 59.37$ & الثاني & $\% 22.89$ & 19 & المفاهيم الأحيائية & 2 \\
\hline$\% 9.37$ & السادس 1 & $\% 3.61$ & 3 & الاحساس و التمتع بالموجودات & 3 \\
\hline$\% 25$ & الر ابع & $\% 9.94$ & 8 & البيئة المحيطة و الو اقع & 4 \\
\hline- & - & - & - & الوعي الجمالي & 5 \\
\hline$\% 6.25$ & 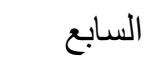 & $\% 2.4$ & 2 & الحفاظ على الكائنات الحية & 6 \\
\hline$\% 28.12$ & الثالث: & $\% 10.84$ & 9 & النظافة العامة & 7 \\
\hline$\% 9.37$ & السادس 2 & $\% 3.61$ & 3 & الجمال و المجتمع & 8 \\
\hline$\% 100$ & الأول & $\% 38.55$ & 32 & اخر اج الكتاب المدرسي & 9 \\
\hline$\% 100$ & & $\% 100$ & 83 & موع & \\
\hline
\end{tabular}

يتضح من النتائج الواردة في الجدول (3) أن كتاب العلوم (الجزء الاول) للصف الثاني الثاني المتوسط قد حقق (83)

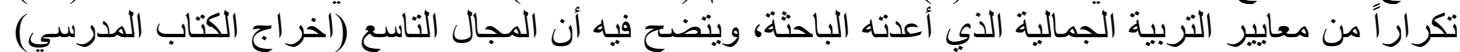

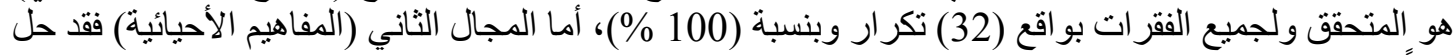

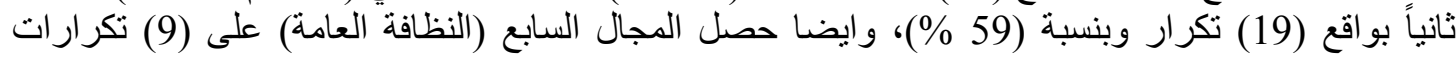

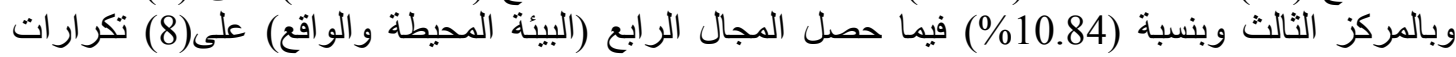




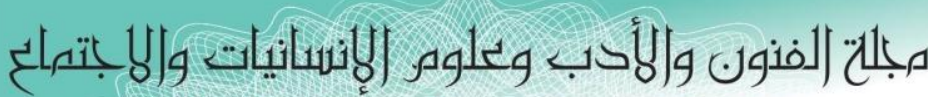
Journal of Arts, Literature, Humanities and Social Sciences

ISSN online: 2414 - 3383

ISSN print: 2616 - 3810
ديسمبر 2019

Volume (46)
العدد (46)

December 2019

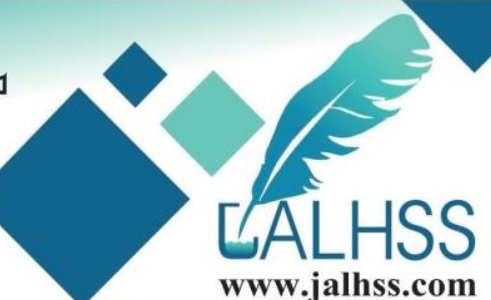

www.jalhss.com

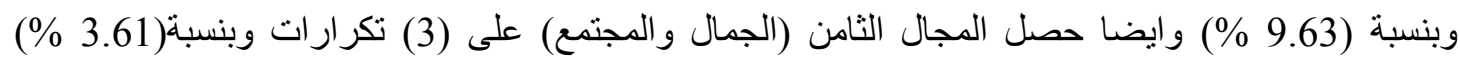

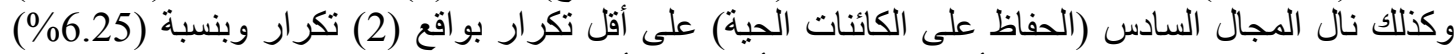

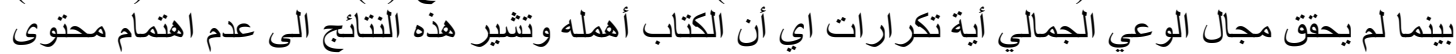

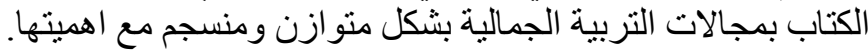

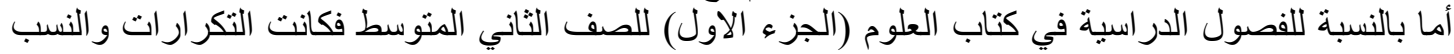

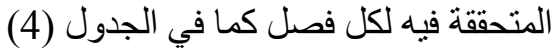

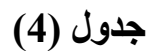

تكرارات ونسب التربية الجمالية في فصول كتاب العلوم (الجزء الاول) للصف الثاني المتوسط

\begin{tabular}{|c|c|c|c|c|}
\hline الترتيب & اللفصلة المئوية & الفصل ع تكر ارات & فصف الثاني المتوسط العلوم (الجزء الاول) & ت \\
\hline 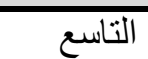 & $\% 3.57$ & 3 & العناصر و التّر ابط الكيميائي & 1 \\
\hline السابع & $\% 7.14$ & 6 & المركبات الكيميائية & 2 \\
\hline الثنامن & $\% 4.76$ & 4 & الصيغ والتفاعلات الكيميائية & 3 \\
\hline 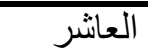 & $\% 2.38$ & 2 & المحاليل & 4 \\
\hline السادس 1 & $\% 8.33$ & 7 & علم التصنيف & 5 \\
\hline 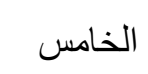 & $\% 9.52$ & 8 & كيف تصنف الكائنات الحية؟ & 6 \\
\hline الأول & $\% 16.66$ & 14 & الكائنات الحية البسيطة & 7 \\
\hline السادس 2 & $\% 8.38$ & 7 & مملكة النبات & $\overline{8}$ \\
\hline الرابع & $\% 11.9$ & 10 & مملكة الحيوان & 9 \\
\hline الثالث & $\% 13.1$ & 11 & البيئة ومكوناتها & 10 \\
\hline الثاني & 14.28 & 12 & التوازن في النظام البيئي & 11 \\
\hline & $\% 100$ & 84 & & 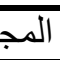 \\
\hline
\end{tabular}

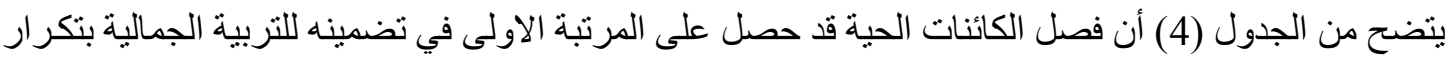

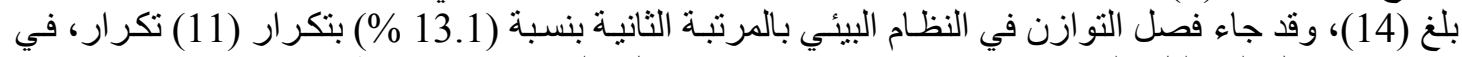

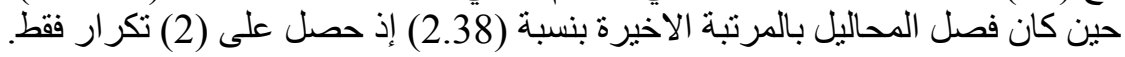

الاستنتاجات: Conclusions

بعد الانتهاء من عرض النتائج وتفسير ها، توصلت الباحثة إلى عدد من الاستنتاجات ومنها الآتي:

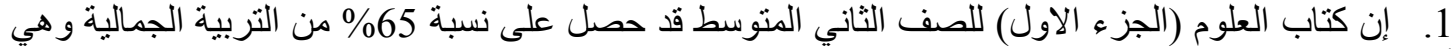

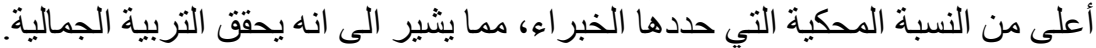

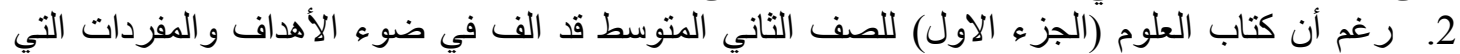

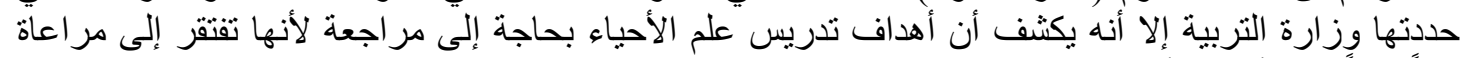

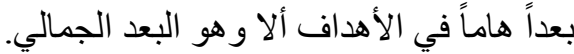
3. ان التربية الجمالية ضرورية في خلق الابداع وتتعكس على حياة الطالب ومجتمعه بثكل ايجابي.

Recommendation : التوصيات

في ضوء النتائج التي توصل الية البحث توصلت الباحثة الى ما يأتي :

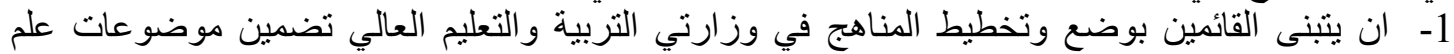
الاحياء لفقرات التربية الجمالية الو اردة في معيار الدراسة وبشكل يحقق التكامل بين هذه الكتب في تغطيتها لها. 


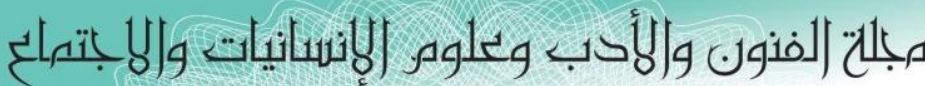

Journal of Arts, Literature, Humanities and Social Sciences

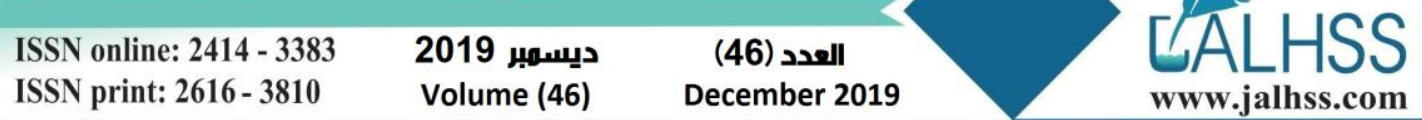

2- ادخال مادة التربية الجمالية ضمن المو اد الدر اسية لمعاهد المعلمين وكليات التربية و التربية الاساسية لما لها من اهمية في اعداد الانسان المتكامل.

3- العمل على اقامة الندوات التعليمية والدورات التدريبية لمدرسي علم الاحياء حول مجالات التربية الجمالية وكيفية تتميتها لدى طلبتهم. 4- العمل على توعية اولياء الامور بماهية التربية الجمالية واهميتها في اعداد الفرد المتكامل في جميع نو احي الحياة. 5- تضمين موضو عات كتب علم الاحياء لآيات من الذكر الحكيم والاحاديث النبوية الثريفة التي تتضمن مفاهيم

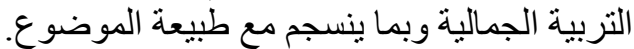
6- بناء وحدة در اسية تتضمن مجالات ومعايير التربية الجمالية في كتب علم الاحباء للمرحلة المتوسطة .

المقترحات: Propositions

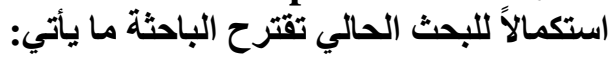

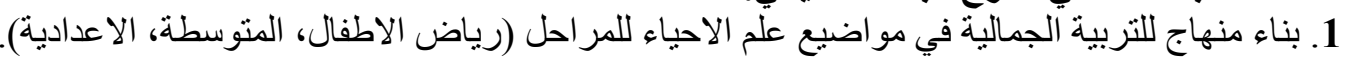

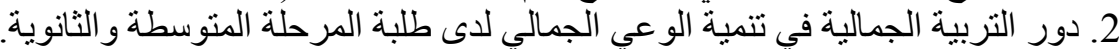
3. تقويم كتب علم الاحياء للمرحلة الاعدادية في ضوع التية التربية الجمالية.

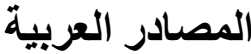

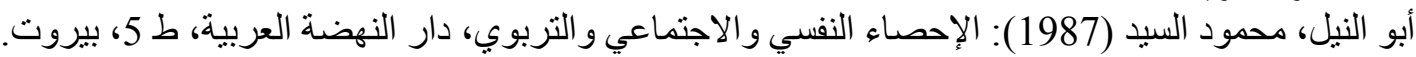
ابو حطب، فو اد (1973): سمات الثخصية و التفضيل الفني ،المجلة الاجنماعية و القومية المباءية المركز القومي للبحوث الاجتماعية والجنائية، المجلد العاثشر يناير القاهرة.

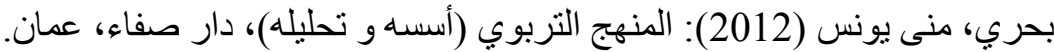

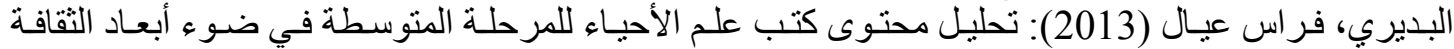

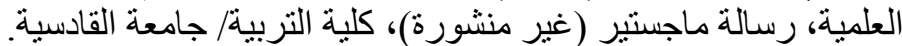

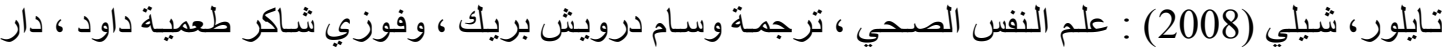

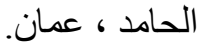
جبار، مروة عبد الحليم صالح (2015): دراسة تحليلية لكتب التربية الأسرية في ضوء إئا الحاجات البيولوجية

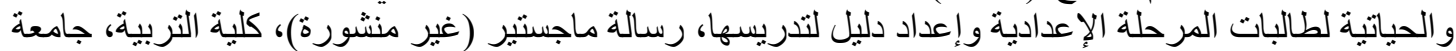
القادسية.

جر ادات، عزت واخرون (1982): مبادئ القياس و التقويم ، المكتبة الوطنية المعاصرة، عمان. جيدوري، صابر (2010): الخبرة الجمالية وابعادها التربوي في فلسفة جون ديوي، مجلة جامعة دمشق، المجلد 26 العدد الثالث. دالين ، ديو بولد ب فان (1985): مناهج البحث في التربية و علم النفس، ترجمة محمد نبيل نوفل ، و آخرون ، السلمان، عبد العالي محمود، وخلف نصار (1987): مقدمة في منهجية تحليل المحتوى، مركز البحوث التربوية

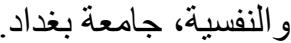

شفيق، محمد (2001): البحث العلمي لإعداد البحوث العلمية، المكتبة الجامعية الإسكندرية، طادية.

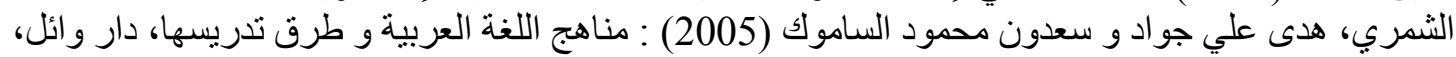
عمان.

الضبع ، محمود (2006) : المناهج التعليمية صناعتهاو تقويمها ، مكتبة الانجلو المصرية ، القاهية القرة.

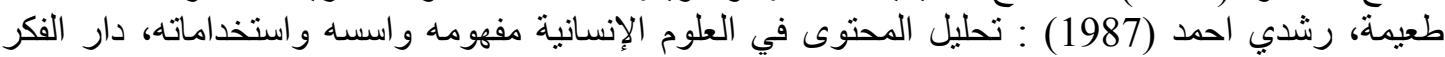
العربي للنشر و التوزيع، القاهرة. طعيمة، رشدي احمد (2011): المنهج المدرسي المعاصر ، أسسـه بنائه، تنظيماتـه تطويره، ط3 ، دار المسيرة للتشر و التوزيع ، عمان. 


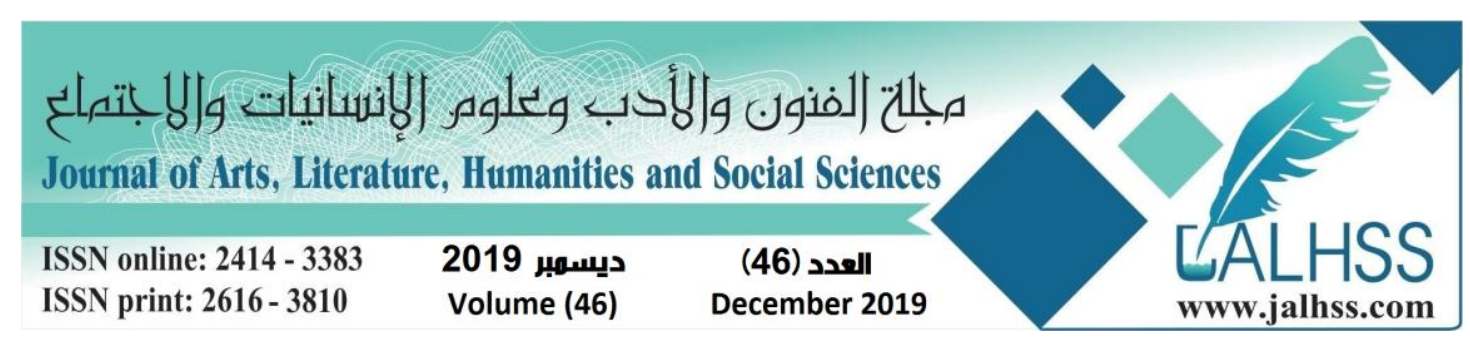

عبد الجو اد، احمد عبد الوهاب، 2001، حتمية تدريب علوم المستقبل في جميع مر احل التعليم، الجمعية المصرية

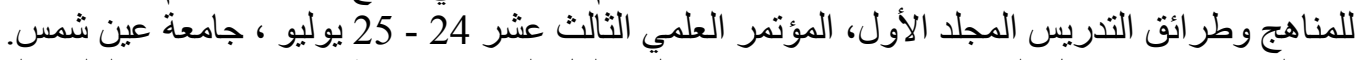

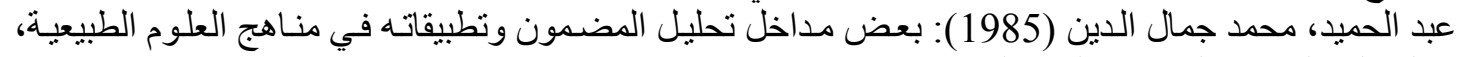

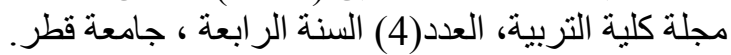

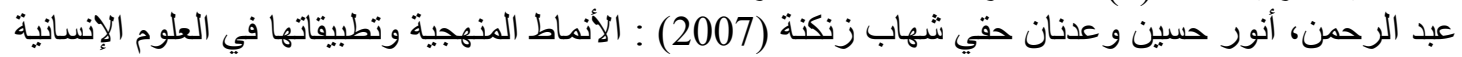

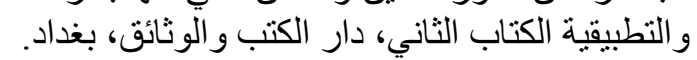
عطية، محسن علي(2010): البحث العلمي في التربية (مناهجه، أدواته، وسائله الاحصائية)، دار المناهج، عمان. الكبيسي، و هيب مجيد (2010): القياس النفسي بين التنظير و التطبيق، العالمية المتحدة للطباعة، بيروت.

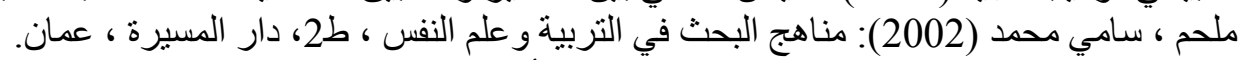

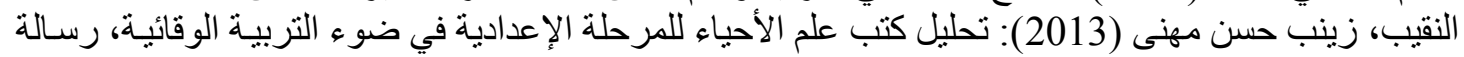
ماجستير (غير منشورة)، كلية التربية/ جامعة القادسية.

\section{References}

1. Abu El-Nile, Mahmoud El-Sayed (1987): Psychological, Social and Educational Statistics, Dar Al-Nahda Al-Arabiya, 5th edition, Beirut.

2. Abu Hatab, Fouad (1973): Personality traits and artistic preference, Social and National Journal, National Center for Social and Criminal Research, vol.

3. Bahri, Mona Younis (2012): The Educational Curriculum (Founded and Analyzed), Dar Safaa, Amman.

4. Al-Budairi, Firas Ayal (2013): Analysis of the content of biology books for the intermediate stage in the light of the dimensions of scientific culture, Master Thesis (unpublished), College of Education / University of Qadisiyah.

5. Taylor, Chile (2008): Health Psychology, Translated by Wissam Darwish Break, and Fawzi Shaker Tamia Dawood, Dar Al-Hamed, Amman.

6. Jabbar, Marwa Abdul Halim Saleh (2015): An analytical study of family education books in the light of the biological and life needs of students in the preparatory stage and the preparation of a guide to teach, Master Thesis (unpublished), College of Education, University of Qadisiyah.

7. Jaradat, Izzat and Others (1982): Principles of Measurement and Evaluation, Contemporary National Library, Amman.

8. Jedouri, Saber (2010): Aesthetic Experience and its Educational Dimensions in the Philosophy of John Dewey, Damascus University Journal, Volume 26, Third Issue.

9. Dalen, De Bold B Van (1985): Research Methods in Education and Psychology, Translated by Mohamed Nabil Nofal, et al., 3rd ed., Anglo-Egyptian Library, Cairo.

10.Al-Salman, Abdul-Ali Mahmoud, and Khalaf Nassar (1987): Introduction to the methodology of content analysis, Center for Educational and Psychological Research, University of Baghdad.

11.Shafiq, Mohamed (2001): Scientific research for the preparation of scientific research, University Library of Alexandria. 


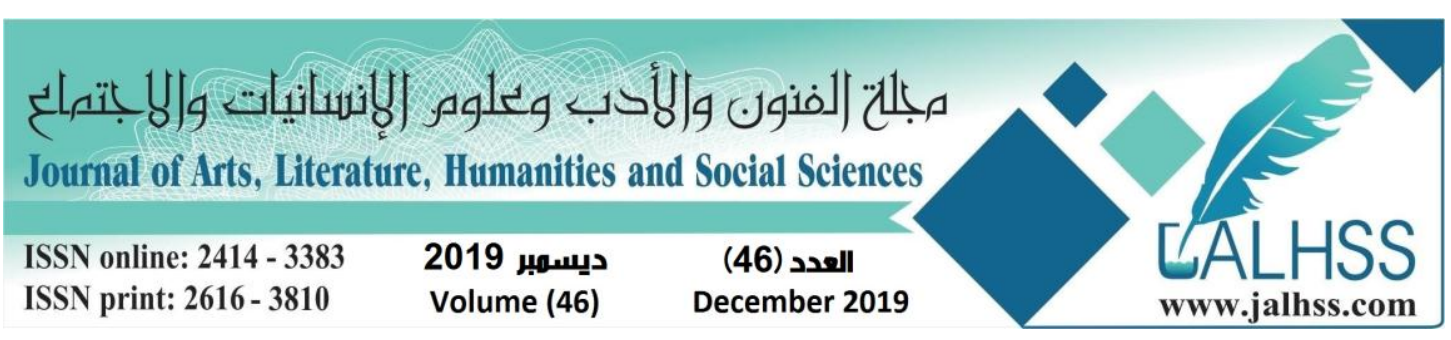

12.Al-Shammari, Huda Ali Jawad and Saadoun Mahmoud Al-Samouk (2005): Curricula and Methods of Teaching Arabic, Dar Wael, Amman.

13.El-Dabaa, Mahmoud (2006): Educational Curriculum Manufacturing and Evaluation, Anglo-Egyptian Library, Cairo.

14.Taima, Roshdy Ahmed (1987): Content Analysis in Humanities: Concept, Bases and Uses, Dar Al Fikr Al Arabi for Publishing and Distribution, Cairo.

15.To'meh, Rushdi Ahmad (2011): The Contemporary School Curriculum, Founded by Its Construction, Development and Organization, 3rd floor, Al-Masirah Publishing and Distribution, Amman.

16.Abdel Gawad, Ahmed Abdel Wahab, 2001, the inevitability of training future science in all stages of education, the Egyptian Association for Curricula and Teaching Methods Volume I, the thirteenth scientific conference 24 - 25 July, Ain Shams University.

17.Abdulhameed, M. Jamal Al-Din (1985): Some Approaches to Content Analysis and its Applications in the Curricula of Natural Sciences, Journal of the College of Education, No. (4) fourth year, Qatar University.

18.Abdul Rahman, Anwar Hussein and Adnan Haqi Shihab Zangana (2007): Methodological Patterns and their Applications in the Humanities and Applied Sciences Book II, Dar al-Kutub wa al-Dawaqaat, Baghdad.

19.Attia, Mohsen Ali (2010): Scientific research in education (curricula, tools, statistical methods), Dar Al-Manaaj, Amman.

20.Al-Kubaisi, Waheeb Majeed (2010): Psychological Measurement between Endoscopy and Application, United International Printing, Beirut.

21.Melhem, Sami Mohammed (2002): Research Methods in Education and Psychology, 2nd edition, Dar Al-Masira, Amman.

22.Captain, Zainab Hassan Mehanna (2013): Analysis of biology books for the preparatory stage in the light of preventive education, Master Thesis (unpublished), College of Education / University of Qadisiyah.

23.Banks, J.A, (1971): "content Analysis of the Black American in Textbook in James, A.B. Teaching Social Studies to Culturally different children, New York, Addison Wesley.

24.Ebel, (1972): Essentials of educational Measurement, New Jersey, Prentice Hall.

25.Festinger, L. (1970): Research methods in the behavioral sciences, American Publishing.

26.Holisti, Ole R. (1969): Content Analysis for the Social Sciences and humanities, Addison -Wesley, New York .

27.Johon, H. M. (2002).Technology and Aesthetic Education: A crucial synthesis. Available from (Eric Document Reproduction service No. EJ 478540).

28.Kerppendroff, K. (1980): Content analysis An Introduction to its methodology, Beverly Hills, CA: Sage, USA.

29.Stmbly, Julian G.\& Kenneth, D. Hopkin (1972): Educational and Psychological Measurement and Evaluation, 5th ed, Englewood cliffs, N. J. prentice Hall.

30.Stone, p. J \& et al., (1966): A computer Approach to Content Analysis, New York. 